\title{
Propagation in Cylindrical \\ Waveguide Containing \\ Inhomogeneous Dielectric
}

The wave equations in the cylindrical waveguide containing inhomogeneous dielectric are $^{1}$

$$
\begin{aligned}
\Delta \times \Delta \times E_{T}-\nabla & \frac{1}{\epsilon} \nabla \cdot \epsilon E_{T} \\
& -\left(\gamma^{2}+\omega^{2} \epsilon \mu\right) E_{T}=0 \\
\epsilon \nabla \times \frac{1}{\epsilon} \nabla \times H_{T} & -\nabla \nabla \cdot H_{T} \\
& -\left(\gamma^{2}+\omega^{2} \epsilon \mu\right) H_{T}=0 .
\end{aligned}
$$

These equations are solved here in two cases: 1) Rectangular waveguide wherein the dielectric constant varies linearly across the broad dimensions, 2) circular waveguide wherein the dielectric constant varies quadratically with the distance from the tube axis. The surface of the waveguide is assumed to be perfectly conductive.

\section{Rectangular Waveguide}

\section{A. LSE-mode ${ }^{2}$}

Putting $E_{x}=0, E_{y}=\cos (m \pi y / 2 b) \cdot X$, $\epsilon=\epsilon_{0}(1-p x)$ in $(1)$ :

$$
\frac{d^{2} X}{d x^{2}}+\left(\gamma^{\prime}-\epsilon^{\prime} x\right) X=0
$$

where $\gamma_{i}=\gamma^{2}+\omega^{2} \epsilon_{0} \mu-(m \pi / 2 b),{ }^{2} \quad \epsilon^{\prime}=p \omega^{2} \epsilon_{0} \mu$ $=\left(2 \pi / \lambda_{0}\right)^{2}\left(n_{1}-n_{2}\right) / a . n_{1}, n_{2}$ are the refraction indexes at $x=0,2 a$, respectively. The solutions of (3) can be written in terms of Bessel function of order $\frac{1}{3},-\frac{1}{3}$. The eigenvalues $\gamma^{\prime}$, which are determined by the boundary condition at $x=0,2 a, E_{y}=0$, are calculated from

$$
\begin{aligned}
& J_{1 / 3}\left(\zeta^{3 / 2}\right) / J_{-1 / 3}\left(\zeta^{3 / 2}\right)=J_{1 / 3}\left(\eta^{3 / 2}\right) / J_{-1 / 3}\left(\eta^{3 / 2}\right) \\
& \text { if } \gamma^{\prime}-2 \epsilon^{\prime} a>0 \\
& =-I_{1 / 3}\left((-\eta)^{3 / 2}\right) / I_{-1} \\
& (-\eta)^{3 / 2} \\
& \text { if } \gamma^{\prime}-2 \epsilon^{\prime} a<0 \\
& \zeta=\left(2 / 3 \epsilon^{\prime}\right)^{2 / 3} \gamma^{\prime}, \quad \eta=\left(2 / 3 \epsilon^{\prime}\right)^{2 / 3}\left(\gamma^{\prime}-2 \epsilon^{\prime} a\right)
\end{aligned}
$$

The eigenvalues $\gamma^{\prime}$, evaluated by the use of asymptotic expansions of the Airy functions, are $^{3}$

$$
\begin{aligned}
& \gamma_{n}^{\prime} \fallingdotseq(n \pi / 2 a)^{2}+\epsilon^{\prime} a \text { if } \quad \epsilon^{\prime} a \ll 1 \\
& 2 \gamma_{n}^{\prime 3 / 2} / 3 \epsilon^{\prime} \fallingdotseq(n-1 / 4) \pi \text { if } \epsilon^{\prime} a>\gamma_{n}{ }^{\prime} . \\
& \text { B. LSM-mode }
\end{aligned}
$$

This mode can be obtained by the similar method as that of LSE-mode.

\section{Circular Waveguide}

In this case, the normal modes are in general combinations of TE and TM modes and are very complicated. Here calculations are made for circularly symmetrical modes ( $\mathrm{TE}_{0 n}$ and $\mathrm{TM}_{0 n}$ modes).

\section{C. $T E_{0 n}$ mode}

Manuscript received April 7, 1965.

1 R. B. Adler, "Waves on inhomogeneous cylindrical structure," Proc. IRE, vol. 40, pp. 339-348, March 1952.

${ }_{2}^{2}$. E. Collin, Field Theory of Guided Waves, New York: McGraw-Hill, 1960.

3 D. E. Kerr, Propagation of Short Radio Waves, Rad. Lab. Ser. 13, New York: McGraw-Hill, 1951. 
Putting $E_{r}=0, \epsilon=\epsilon_{0}\left(1-q r^{2}\right)$ in (1), the equation for $E_{\phi}$ becomes

$$
\begin{aligned}
\frac{d^{2} E_{\phi}}{d r^{2}} & +\frac{1}{r} \frac{d E_{\phi}}{d r} \\
& +\left(\gamma^{\prime \prime}-\epsilon^{\prime \prime} r^{2}-\frac{1}{r}\right) E_{\phi}=0
\end{aligned}
$$

where

$$
\begin{aligned}
& \gamma^{\prime \prime}=\gamma^{2}+\omega^{2} \epsilon_{0} \mu, \\
& \epsilon^{\prime \prime}=q \omega^{2} \epsilon_{0} \mu=2\left(2 \pi / \lambda_{0}\right)^{2}\left(n_{0}-n_{3}\right) / R^{2} .
\end{aligned}
$$

$n_{0}, n_{3}$ are the refraction indexes at $r=0$, $R$, respectively. Equation (6) can be put in Whittaker's standard form of confluent hypergeometric equation by the following transformation: $:^{4}$

$$
z=\sqrt{\epsilon^{\prime \prime}} r^{2}, \quad Y=E_{\phi} / r, \quad \kappa=\gamma^{\prime \prime} / 4 \sqrt{\epsilon^{\prime \prime}}
$$

and we obtain

$$
\frac{d^{2} Y}{d r^{2}}+\left(-\frac{1}{4}+\frac{\kappa}{z}\right) Y=0 .
$$

The solutions of (6) are given in terms of the Whittaker functions:

$$
E_{\phi}=\left(1 / \epsilon^{\prime \prime 1 / 4} r\right) M_{\kappa, 1 / 2}\left(\sqrt{\epsilon^{\prime \prime}} r^{2}\right)
$$

and the eigenvalues $\gamma^{\prime \prime}$, which satisfy $M_{\mathrm{k}, 1 / 2}\left(\sqrt{\epsilon^{\prime \prime}} R^{2}\right)=0$, are calculated from

$$
\begin{array}{r}
\sqrt{\epsilon^{\prime \prime}} R^{2} \fallingdotseq\left(\sqrt{\epsilon^{\prime \prime}} / \gamma_{n}{ }^{\prime \prime}\right) j_{1, n}{ }^{2}\left(1+\left(\epsilon^{\prime \prime} / 3 \gamma_{n}{ }^{\prime \prime 2}\right) j_{1, n}{ }^{2}\right), \\
\text { if } \sqrt{\epsilon^{\prime \prime}} R^{2} \ll 1
\end{array}
$$

where $j_{1, n}$ is the $n$th zero of $J_{1}(x)$,

$$
\begin{aligned}
& \left(\sqrt{\epsilon^{\prime \prime}} R^{2}\right)^{-2 \kappa_{n}} \exp \left(\sqrt{\epsilon^{\prime \prime}} R^{2}\right) \\
= & -\Gamma\left(1-\kappa_{n}\right) / \Gamma\left(1+\kappa_{n}\right), \text { if } \overline{\sqrt{\epsilon^{\prime \prime}} R^{2}>4 n .\left(9^{\prime}\right)}
\end{aligned}
$$

When $\sqrt{\epsilon^{\prime \prime}} R^{2}$ is large, $\gamma^{\prime \prime}$ approaches to an integer and is written as $\gamma_{n}^{\prime \prime}=n+\Delta$. Then

4. Erdelyi, Higher Transcendental Functions, vol. 1. New York: McGraw-Hill, 1953. $\left(9^{\prime}\right)$ becomes

$$
\begin{aligned}
\left(\sqrt{\epsilon^{\prime \prime}} R^{2}\right)^{-2 n} \exp \left(\sqrt{\epsilon^{\prime \prime}} R^{2}\right) & \fallingdotseq \frac{(-1)^{n-1}}{n !(n-1) ! \Delta}, \\
n & =1,2, \cdots \quad(9
\end{aligned}
$$

and $(1 / z)^{1,2} M_{\kappa, 1 / 2}(z)$ approaches $z^{1 / 2} \exp$ $(-z / 2) L_{n-1}^{1}(z)$.

\section{D. $T M_{0 n}$ mode.} (2):

The wave equation for $H_{\phi}$ follows from

$$
\begin{array}{r}
\frac{d^{2} H}{d r^{2}}+\frac{1}{r} \frac{d H_{\phi}}{d r}-\frac{1}{\epsilon} \frac{d \epsilon}{d r}\left(\frac{d H_{\phi}}{d r}+H_{\phi}\right) \\
+\left(r^{\prime \prime}-\epsilon^{\prime \prime} r^{2}-\frac{1}{r^{2}}\right) H_{\phi}=0 .
\end{array}
$$

This equation is solved by the perturbation method and the first approximation of the solution is

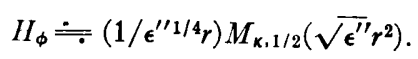

The eigenvalues $\gamma^{\prime \prime}$, which satisfy

$$
(d / d z) M_{\kappa, 1 / 2}(z)=0 \text { at } z=\sqrt{\epsilon^{\prime \prime}} R^{2},
$$

are calculated from

$$
\begin{aligned}
& \sqrt{\epsilon^{\prime \prime}} R^{2} \fallingdotseq\left(\sqrt{\epsilon^{\prime \prime}} / \gamma_{n}{ }^{\prime \prime}\right) j_{0, n}{ }^{2}, \text { if } \sqrt{\epsilon^{\prime \prime}} R^{2} \ll 1(12) \\
&\left(\sqrt{\epsilon^{\prime \prime}} R^{2}\right)^{-2 n} \exp \left(\sqrt{\epsilon^{\prime \prime}} R^{2}\right) \fallingdotseq \frac{(-1)^{n}}{n !(n-1) ! \Delta}, \\
& \text { if } \sqrt{\epsilon^{\prime \prime}} R^{2}>4 n .\left(12^{\prime}\right)
\end{aligned}
$$

From the results of the preceding calculations, we conclude that, 1) if the degree of inhomogeneity is low $\left(\epsilon^{\prime} a \ll 1, \sqrt{\epsilon^{\prime \prime}} R^{2} \ll 1\right)$, the configurations of the preceding modes do not differ much from those of homogeneous waveguides, 2$)$ if the degree of inhomogeneity is high $\left(\epsilon^{\prime} a>\gamma_{n}{ }^{\prime}, \sqrt{\epsilon^{\prime \prime}} R^{2}>4 n\right)$, these solutions turn into the surface waves, and the field concentrates in the region having larger dielectric constant and decays exponentially away from this region, as shown in Fig. 1.

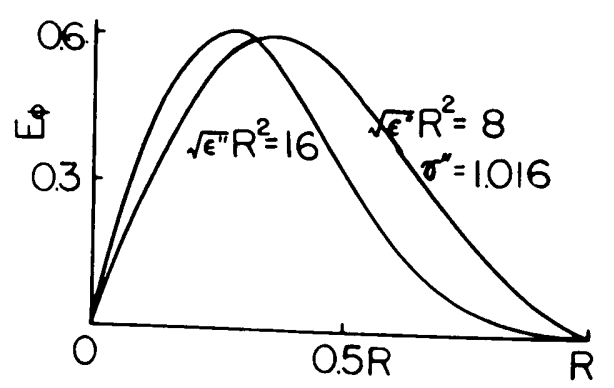

Fig. 1. Field distribution of TE 01 mode.

These surface wave modes may propagate with very low attenuation regardless of the wall condition.

In the case when the inhomogeneity of the dielectric is caused by the temperature stratification of the air in the waveguides, the refraction index varies with the temperature as $n_{1}-n_{2}=0.93 \times 10^{-6}\left(T_{2}-T_{1}\right)$, and the trapping conditions for the lowest modes, i.e., $\epsilon^{\prime} a>\gamma_{\mathbf{l}^{\prime}}, \sqrt{\epsilon^{\prime \prime}} R^{2}>4$, can be rewritten with the temperature as $a / \lambda_{0}>10^{2} / \sqrt{T_{2}-T_{1}}$ for rectangular waveguide, $R / \lambda_{0}>5$ for rectangular waveguide,

$$
R / \lambda_{0}>5 \times 10^{2} / \sqrt{T_{3}-\overline{T_{0}}}
$$

$\times 10^{2} / \sqrt{T_{3}-T_{0}}$ for circular waveguide.

With these simplified models, we may examine the influence of the temperature stratification of the air in the oversize waveguides for transmission of submillimeter and shorter wavelength region. ${ }^{5}$

R. YAMADA

K. WATANABE

Dept. of Electronics

Shizuoka University Hamamatsu City, Japan

${ }^{5} \mathrm{G}$. Goubau and J. R. Christian, "Some aspects of beam waveguide for long distance transmission at optical frequencies," IEEE Trans. on Microwave Theory and Techniques, vol. MTT-12, pp. 212-220,
March 1964. 